\title{
The Measurement Method of Population Dynamics and
}

\section{New Measurement Index}

\author{
Chengguo Jiang \\ College of Life Science, Shihezi University \\ Xinjiang 832003, China \\ E-mail: jiangchengguo206@sina.com
}

Tong Liu

College of Life Science, Shihezi University

Xinjiang 832003, China

E-mail: liutong1968@yahoo.com.cn

Dan Wang

College of Life Science, Shihezi University

Xinjiang 832003, China

\begin{abstract}
It is hard to describe the population dynamics by specific numbers because there are many influencing factors, such as accepting pollination, nutrition source, predator, disease and breeding way, and so on. The paper adopts the method of appropriate concentric circle and new population dispersing index for population dynamics study.

Its main purposes includes: (1) the comparison for same area in different years; (2) the comparison for same community in different areas; (3) the comparison for the advantages and disadvantages in different population in same community. Meanwhile, the paper points out the advantages and disadvantages of this index, probe into the further optimized measures and try to study the population dynamics scientifically and rationally.

Keywords: Index of scale, Concentric circle quadrat, Population dispersion index, Weighted disposal

\section{Introduction}

Plant population is dynamic, so there is certain degree of difficulty for its study. Generally speaking, once the plant survives, it has no removability and its reproduction is influenced by both the external and the internal factors. Among the evaluation system of the plant population indications, the quadrat position of the plant is vital. The present studies of population dynamics mainly consider the genetic structure, spatial structure, age structure and size structure, which have some limitation because they do not unity the sample plot. The values of statistical equation in population statistics stress in the quantity change and can not forecast effectively the future development tendency of population. In view of the above reasons and the results observed by myself for many years, I proposed the method of concentric circle quadrat to carry on the sampling and the new evaluation index, that is, population dispersion index. This quadrat and the dispersion index can be combined well, and unifies the sample plot position of each plant to describe the future trend of population scientifically.
\end{abstract}

\section{Design of survey quadrat}

Concentric circle quadrat is a kind of quadrat made of a group of concentric circles which takes the distribution centre 
of population as the center of circle, a group of arithmetic progressions as the radius. The grid method and transect method are two common methods for survey of population and community dispersion because they are easy to operate and for statistics. However, these two methods are different to discover the center of sample plot and to calculate precisely the concrete position of each plant.

Concentric circle quadrat can determine the differences and units of radiuses between two neighboring circles according to different need. All neighboring radius differences must be equal, and this difference value should be the radius of minimum quadrat circle so that all the radiuses of quadrats circles form to be a group of arithmetic progressions. The detailed quadrat mold illustrations are as follows:

As shown in Picture 1, "S1,S2,S3 ..... Sn" respectively represent the area of Quadrat $\mathrm{i}$, which require $\mathrm{S}_{1}=\pi \mathrm{r}_{1}{ }^{2}$, $\mathrm{S}_{2}=\pi \mathrm{r}_{2}{ }^{2}-\pi \mathrm{r}_{1}{ }^{2}, \mathrm{~S}_{3}=\pi \mathrm{r}_{3}{ }^{2}-\pi \mathrm{r}_{2}{ }^{2} \ldots \ldots . \mathrm{S}_{\mathrm{n}}=\pi \mathrm{r}_{\mathrm{n}}{ }^{2}-\pi \mathrm{r}_{\mathrm{n}-1}{ }^{2}$. Among them, the unit should be determined by radius. $\pi$

" $\mathrm{N}_{1}, \mathrm{~N}_{2}, \mathrm{~N}_{3} \ldots \ldots \mathrm{N}_{\mathrm{n}}$ " in Picture 2 respectively represent the surveyed plant number of Quadrat $\mathrm{n}$.

" $\mathrm{L}_{1}, \mathrm{~L}_{2}, \mathrm{~L}_{3} \ldots \ldots . \mathrm{L}_{\mathrm{n}}$ " in Picture 3 respectively represent the distance between the center of Quadrat $\mathrm{n}$ and that of sample plot. Among them, the starting point of $\mathrm{L}$ is the center of sample plot and the end points of $\mathrm{L}$ are the center line between the Circle $n$ and Circle $n-1$.

\section{Definition of population disperse index and formula}

\subsection{Conception of population dispersion index}

The population dispersion index is the indicator of the quantity, reproductive ability, dispersion condition in some area or space for the plant population and the survival potential of this species. It is the index to describe the dispersion condition of some species, the degrees of outward expanding and increasing and inward contracting of this species. Especially for the community of endangered species or the growth condition of local community, it can obtain the annual changing conditions and good or bad local communities according to the comparison of survey datum and then forecast their development tendencies so that propose the relative scientific measures in good season for population protection.

\subsection{Population dispersion index}

$$
\mathrm{Dp}=\sum_{i=1}^{n}\left(\frac{L_{i}}{r_{n}} \frac{S_{1} N_{i}}{S_{i}}\right)
$$

As is shown in the above formula, "i" represents Quadrat $i$, " $n$ " represents the total number of quadrat, "Dp" represents population dispersion index, $L_{i}$ represents the distance between center of Quadrat i and center of sample plot, "Rn" represents the radius of maximum quadrat circle, "S1" represents the area of minimum quadrat, "Si" represents the area of Quadrat i and "Ni" represents the number of this species in Quadrat i..

\subsection{Further disposal of population dispersion index}

The population belongs to an aggregate and the perennial plants' age structure decided its reproductive capacity, which is very a big influence to the local community. To eliminate this influence, we could adopt the weighted disposal for the plant numbers. For the better reproductive individual, adding a parameter before Ni. For example, one plant is 1.2 times as better as other plant, we could calculate it as 1.2 plants. However, this weighted disposal is not advocated except there is a full comprehend to the plant.

\subsection{Application of population dispersion index}

Population dispersion index is suitable for the survey of animal, plant and microorganism. Detailed Applications include:

(1) The comparison for same area in different years. If $\mathrm{Dp}_{\mathrm{N}} / \mathrm{Dp}_{\mathrm{N}-1}>1$, this population belongs to Increasing Type; if $D p_{\mathrm{N}} / \mathrm{Dp}_{\mathrm{N}-1}=1$, this population belongs to Steady Type; if $\mathrm{Dp}_{\mathrm{N}} / \mathrm{Dp}_{\mathrm{N}-1}<1$, this population belongs to Decline Type.

(2) The comparison for same community in different areas. For example, there are two district of A and B. If the dispersion index of A is bigger than that of B, District A is better than District B; otherwise District A is worse than District B.

(3) The comparison for the advantages and disadvantages in different population in same community. Generally speaking, the bigger dispersion index means the better advantages of this species.

The above applications must be based on the same sample plot and unit for comparison.

\subsection{Potential error and correction}

The potential error of population dispersion index may be resulted from two points: (1) the setting of quadrat area; (2)the 
determination of quadrat center. Considering the above points must be depend on human's subjective judgement, the size and location of the quadrat should be determined according to the density, the distribution, the terrain and the reproduction characteristic in the survey process. The center of circle must be located in the center of population distribution, and it should adopt the same center for same population in different years. For the comparison of different populations, it can also adopt the same quadrat.

\section{Discussion}

There are many influencing factors for population dynamics, such as accepting pollination, nutrition source, predator, disease and breeding way, and so on. Meanwhile, the plants are influenced largely by its own population density and the seed dissemination way and the location of the species is just one factor, however, it is a distinctive method comparing with the previous statistical methods. This research technique and the statistical method have higher rationality and the comparison using this population dispersion index can have more advantages. Of course, like the grid method and transect method this survey method may has some difficulty in the operation and it may depend on the personal experience, so the selection of quadrat center and the circle space should referred to various factors.

\section{References}

Gilpin, M. (1972). Enriched predator-prey systems: theoretical stability. Science, 177, 902-904.

Hastings, A. (2001). Transient dynamics and persistence of ecological systems. Ecol. Lett. 4, 215-220.

Holmes, E.E., Lewis, M.A., Banks, J.E., Veit, R.R., (1994). Partial differential equations in ecology: spatial interactions and population dynamics. Ecology, 75, 17-29.

Kot, M. (2001). Elements of Mathematical Ecology. Cambridge Univeristy Press, Cambridge, UK.

Kullback, S., (1959). Information Theory and Statistics. John Wiley \& Sons, New York.

Loehle, C., Li, B.-L., (1996). Habitat destruction and the extinction debt revisited. Ecol. Appl. 6, 784-789.

Ma K P. Measurement of biodiversity. In: Qian Y Q ed. Principle and methods of biodiversity studies*. Beijing: Chinese Scientific \& Technological Press. (1994). 2(3): 162-168.

Perter Turchin, George O.Batzli. Availability of Food and the Population Dynamics of Arvicoline Rodents. The Ecological Society of American, (2001). 82(6): 1521-1534.

Perter Turchin. Complex Population Dynamics: A Theoretical Empirical Synthesis. New Jersey: Princetor University Press, (2003).

Yoshizawa T. Stability Theory By Liapunov Second Method Tokyo: The Mathematical Society of Japan ,1966. 59-61.

Zhang Jintun. Quantitative Ecology. Science Press. (2003). 94-96.

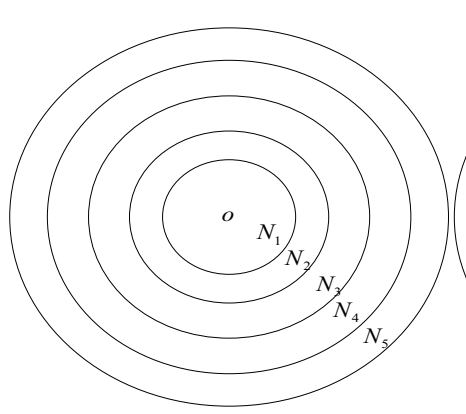

Figure 1

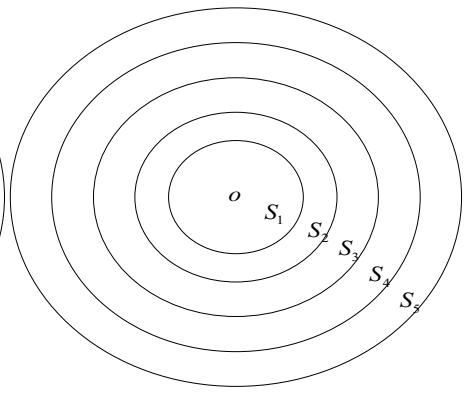

Figure 2

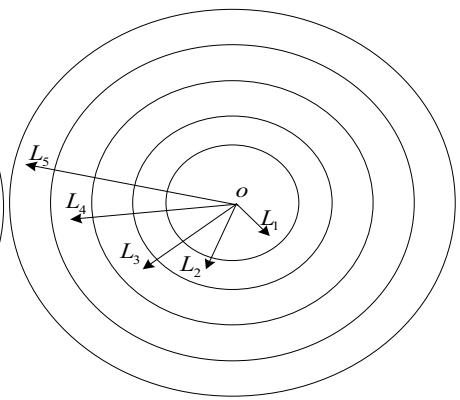

Figure 3 